\title{
Investigation of Wave Processes in a Rectangular Region with Discontinuous Boundary Conditions
}

\section{Nurgali ASHIRBAYEV*, Zhansaya ASHIRBAYEVA**, Gaukhar SARSENOVA***, Raina BEKMOLDAYEVA****, Zhuldyz NURMAGANBETOVA*****, Peruza DUISEBAYEVA*******}

*M. Auezov South Kazakhstan State University, Tauke Khan Avenue 5, 160012 Shymkent, Kazakhstan, E-mail: ank_56@mail.ru

**M. Auezov South Kazakhstan State University, Tauke Khan Avenue 5, 160012 Shymkent, Kazakhstan, E-mail: saya_270681@mail.ru

***M. Auezov South Kazakhstan State University, Tauke Khan Avenue 5, 160012 Shymkent, Kazakhstan, E-mail: sarsen_2006@mail.ru

****M. Auezov South Kazakhstan State University, Tauke Khan Avenue 5, 160012 Shymkent, Kazakhstan, E-mail: raina_b@mail.ru

*****M. Auezov South Kazakhstan State University, Tauke Khan Avenue 5, 160012 Shymkent, Kazakhstan, E-mail: juju456@mail.ru

******M. Auezov South Kazakhstan State University, Tauke Khan Avenue 5, 160012 Shymkent, Kazakhstan, E-mail: peruza_69@mail.ru

cross'ref http://dx.doi.org/10.5755/j01.mech.24.5.20102

\section{Introduction}

The study of propagation of dynamic perturbations in an elastic body of finite dimensions, taking into account a number of attenuating factors (discontinuities in boundary conditions, holes, cavities, cutouts, etc.), has not only theoretical but also applied value determined by requests of engineering practice.The solution of the corresponding specific problems and the study of non-stationary wave fields make it possible to reveal the physical consequences of various kinds, to assess the strength and reliability of work of entire construction. When constructing analytic solutions of plane problems for domains of finite dimensions, there arise insurmountable difficulties in mathematical modeling of the processes of multifold reflection, diffraction, and interference of wave perturbations.

The solution of boundary-value problems for such domains, as a rule, requires the development of effective non-standard or modified numerical algorithms also. In connection with rapid growth of modern computing facilities and modern information technologies, there is also a necessity for their use for solving problems of these kinds and obtaining working programs for personal computers which allow to calculate a particular state of objects in concrete problems of practical direction [cf. 1-7].The purpose of this study is to develop a difference method of spatial characteristics for solving plane problems of dynamic elasticity with discontinuous boundary conditions.

\section{Mathematical formulation of the problem}

Let us consider the basic equations of the dynamic elasticity theory for a homogeneous isotropic elastic body in the Cartesian coordinate system. For convenience the axes of the Cartesian coordinate system are denoted by $O x_{i}(i=1,2)$, and the time by $t$. Components of stresses ten- sor are denoted through $\sigma_{i j}\left(x_{1}, x_{2}, t\right)$, of deformations tensor-through $\varepsilon_{i j}\left(x_{1}, x_{2}, t\right) \quad(i, j=1,2)$, and of displacements tensor - through $u_{i}\left(x_{1}, x_{2}, t\right) \quad(i=1,2)$.

Equations of motion and Hooke's law for a homogeneous isotropic elastic medium can be written in the form:

$$
\begin{aligned}
& \sigma_{i j, j}=\rho \partial^{2} u_{i} / \partial t^{2} \quad(i=1,2), \\
& \sigma_{i j}=\lambda \theta \delta_{i j}+2 \mu \varepsilon_{i j}
\end{aligned}
$$

where: $\rho$ is density of medium material, $\lambda, \mu$ are the Lamé constants, $\delta_{i j}$ is the Kronecker symbol, $\theta$ is the volume deformation equaled to:

$$
\theta=\varepsilon_{11}+\varepsilon_{22}+\varepsilon_{33} .
$$

Comma with index means partial derivative with respect to the corresponding argument. The summation is performed over $j(j=1,2)$.

Following [8], non - dimensional variables were introduced using the formulas:

$$
\begin{aligned}
& \vec{t}=\frac{t c_{1}}{b} ; \quad \vec{x}_{i}=\frac{x_{i}}{b} ; \quad v_{i}=\frac{1}{c_{1}} \frac{\partial u_{i}}{\partial t}, \quad(i=1,2) \\
& p=\frac{\sigma_{11}+\sigma_{22}}{2 \rho c_{1}^{2}} ; \quad q=\frac{\sigma_{11}-\sigma_{22}}{2 \rho c_{1}^{2}} ; \\
& \tau=\frac{\sigma_{12}}{\rho c_{1}^{2}} ; \quad \gamma=\frac{c_{1}}{c_{2}},
\end{aligned}
$$

where: $b$ is the characteristic length, $c_{1}, c_{2}$ are the velocities of the expansion and the shear waves, $\sigma_{11}, \sigma_{22}, \sigma_{12}$ are the components of the stress tensor, and $\gamma$ is a constant 
parameters. Hereinafter, the line over non -dimensional parameters are omitted.

Then under the conditions of plane deformation, the wave process at the interior points of the rectangular strip is described as a system of equations for the velocities of displacements $v_{1}, v_{2}$ and three linear combinations of the stress tensor components $p, q, \tau[8]$ :

$$
\begin{aligned}
& v_{1},{ }_{t}-p,_{1}-q,_{1}-\tau,,_{2}=0 \text {; } \\
& v_{2},{ }_{t}-p,_{1}-q,_{2}-\tau_{, 1}=0 \text {; } \\
& \gamma^{2}\left(\gamma^{2}-1\right)^{-1} p,,_{t}-v_{1},{ }_{1}-v_{2},_{2}=0 \text {; } \\
& \gamma^{2} q,{ }_{t}-v_{1},{ }_{1}+v_{2}, 2=0 \text {; } \\
& \gamma^{2} \tau,_{t}-v_{1,2}-v_{2,1}=0 \text {. }
\end{aligned}
$$

Comma with index means partial derivative with respect to the corresponding argument. This formulation is chosen because such systems have been studied quite well by now, and, moreover, when choosing the velocities of displacements and stresses as dependent variables, the derivatives are excluded from the boundary conditions.

The plane deformation of elastic body with rectangular cross-section is investigated. The cross-section has dimensions $0 \leq x_{1} \leq l, \quad-L \leq x_{2} \leq L$ (Fig. 1). The boundary-value problem, formulated for the resolving equations (4), assumes that at the initial moment of time $t=$ 0 the body is at rest:

$$
\begin{aligned}
& v_{1}\left(x_{1} ; x_{2} ; 0\right)=v_{2}\left(x_{1} ; x_{2} ; 0\right)=p\left(x_{1} ; x_{2} ; 0\right)= \\
& =q\left(x_{1} ; x_{2} ; 0\right)=\tau\left(x_{1} ; x_{2} ; 0\right)=0 .
\end{aligned}
$$

At any other moment of time $t>0$ on the boundary $x_{1}=0,-L \leq x_{2} \leq L$ an uniformly distributed load operates varying with time according to the law of the continuously differentiable function $f(t)$, which monotonically increases to the maximum value $f\left(t_{0}\right)$ at the beginning, and then decreases monotonically:

$$
v_{1}=f(t), \quad v_{2}=0 \text { at } x_{1}=0, \quad-L \leq x_{2} \leq L .
$$

The lateral edges $\left|x_{2}\right|=L$ of the strip are stressfree:

$$
p-q=0, \quad \tau=0 \text { at }\left|x_{2}\right|=L, \quad 0 \leq x_{1} \leq l .
$$

On the lower edge $x_{1}=l$ of the strip inhomogeneous boundary conditions are considered:

$v_{1}=v_{2}=0 \quad \forall\left|x_{2}\right| \leq x_{2}^{0}$ and $p+q=\tau=0 \quad \forall\left|x_{2}\right| \geq x_{2}^{0}$,

where: $x_{2}^{0}$ is some constant number satisfying the requirement $\left|x_{2}^{0}\right| \leq l$.

To compare the results of the problem solutions, we consider additional problem, in which it is assumed that the boundary $x_{1}=l$ of the strip is rigidly fixed, i.e.

$$
v_{1}=v_{2}=0 \text { at } x_{1}=l,\left|x_{2}\right| \leq L
$$

The boundary conditions (8) correspond to the conditions under which the middle part $x_{1}=l$ of the boundary is rigidly fixed, and its lateral sections are free of stresses. They mimic the work conditions of a single-bearing construction. Under the accepted loading (6), in the body a complex process of propagation of longitudinal in the directions of the axes $x_{1}, x_{2}$ and transverse waves arises, which after a while (depending on the size and speed of propagation of perturbations) begin to interfere. It is necessary to investigate the wave motion inside the considered body at $t$ $>0$.

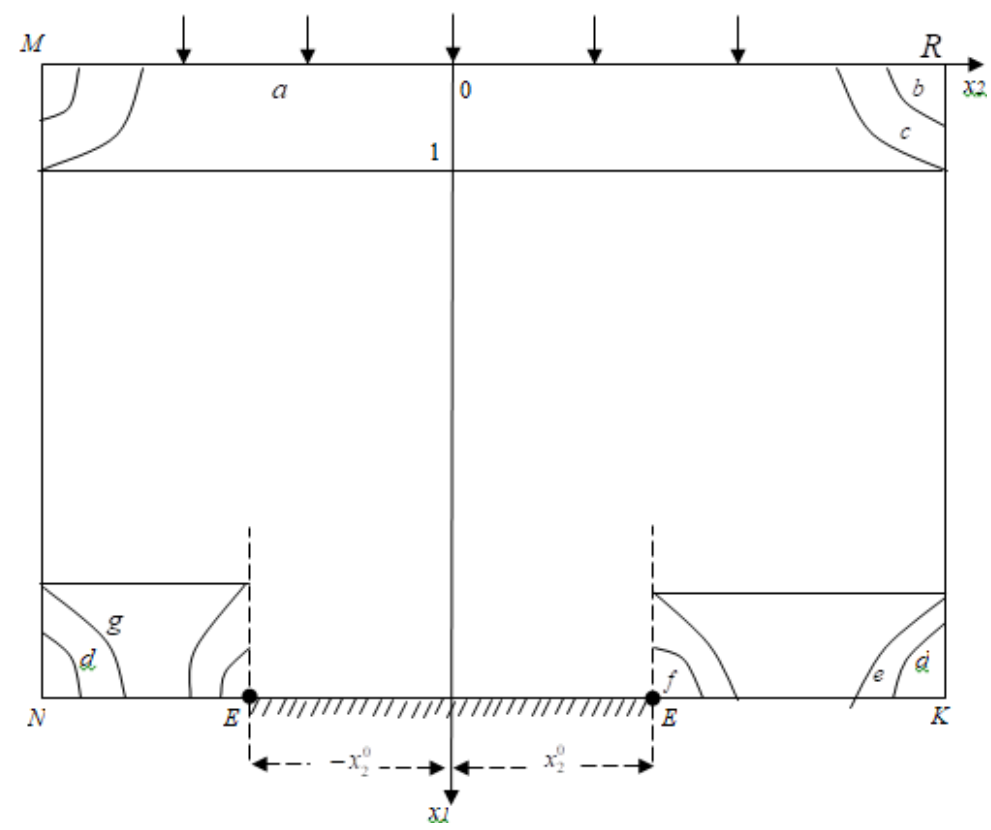

Fig. 1 Region of the research

The solution of the equations system (4) for initial (5) and boundary conditions (6) - (8) is found out by the difference method of spatial characteristics. The algorithm for solving Eq. (4) on the basis of spatial characteristics 
method was developed in [8] for interior, boundary and corner points and the corresponding calculated finite-difference relations were obtained. It is based on the finite-difference relations obtained by integrating the equations (4) along bicharacteristics and written at nodal points, into which the entire investigated region is divided (Fig.1). When consideration of the inhomogeneous boundary conditions (8), for each of the corner points $N\left(x_{1}=l, x_{2}=-L\right), K$ $\left(x_{1}=l, x_{2}=L\right)$, the law of twoness of tangential stresses is fulfilled. This circumstance reduces the number of given conditions at the corner point by one, and in order to obtain a closed system of equations, it is necessary to obtain one additional equation for the corner $N\left(x_{1}=l, x_{2}=-L\right)$ and $K$ $\left(x_{1}=l, x_{2}=L\right)$ respectively. The method of obtaining of additional equations for each of the corner points of a rectangular region was considered in [8]. However, all the finite-difference relations obtained can be used only in regions with a continuous change of all incoming parameters.

On the boundary $x_{1}=l$ at points of type $E$ (Fig.

1) the calculated relations have singularities. In the problem under study the points $\left(x_{1}=l,\left|x_{2}\right|=x_{2}^{0}\right)$, lying on the boundary $x_{1}=l$ of the strip, are singular because of jumplike change in the boundary conditions. In the work [8] singularity of the solution for the region, that takes place in the problem under study, is noted and for such points a calculation method is not developed. In addition, the unknown functions and their first and second derivatives at the boundary nodal points of type $E$ of the strip suffer a discontinuity of the first kind in the direction of the axis $x_{2}$. In connection with this, at the points of their conjugation, where the boundary conditions change jump-like, the method was proposed for obtaining resolving equations for finding the unknown functions $v_{1}, v_{2}, p, q, \tau$ [9]. It should be noted that when approximation of the first and second derivatives of the unknown functions in the direction of the axes $x_{1}, x_{2}$, at the boundary nodal points of the strip a nine-point difference scheme is usually used [8]. In the presence of singular points of type $E$ at the boundaries the nine-point scheme may turn out to be insufficient for approximation of the first and second derivatives of the desired functions. Therefore, in the proposed approach, a 15-point difference scheme is used to approximate the first and second derivatives of the unknown functions at singular boundary points of type $E$ [9].

Thus, the numerical algorithm for solving the nonstationary elasticity problem has been constructed at singular points, in which the incoming parameters suffer a discontinuity of the first kind. On the basis of this numerical algorithm unified calculation program has been developed in the Fortran language for personal computers.

\section{Analysis of calculation results}

On elastic body in the form of rectangular strip a square grid is plotted, at the nodes of which the values of the velocity components $v_{1}, v_{2}$, displacements and stresses $p, q$, $\tau$ are determined. It is assumed that the boundaries of the body coincide with the line of nodes of the square grid that covers the region under investigation (Fig. 1).

The computational process is carried out in time steps. The time step $k$ is selected in accordance with the necessary stability conditions of used explicit finite difference scheme [6].

$$
\left(\frac{k}{h}\right)^{2} \leq \min \left\{\frac{\gamma^{2}}{\gamma^{2}+1}, \frac{\gamma^{2}}{2\left(\gamma^{2}-1\right)}\right\}
$$

In this way, the values of the unknown quantities are calculated at any point of the rectangular strip at the time moment $t=t_{0}=k \Delta t$. To obtain the results in the next step with respect to the time $t=(k+1) \Delta t$ it is sufficient to take the found values as the initial data and repeat the calculations. For numerical realization of the developed finite-difference scheme and solving non-stationary problems of mechanics of a deformable solid the technique and calculation algorithm have been created and on their basis the complex of computational programs in the Fortran-90 language has been developed for high-speed personal computers.

Numerical results are given for the rectangular region $0 \leq x_{1} \leq 100 h,\left|x_{2}\right| \leq 100 h$. Body material has the following characteristics: elastic modulus $E=200 \mathrm{GPa}$; Poisson's coefficient $v=0.3$; density $\rho=7.9 \cdot 10^{3} \mathrm{~kg} / \mathrm{m}^{3}$, $c_{1}=5817 \mathrm{~m} / \mathrm{sec}, c_{2}=3109 \mathrm{~m} / \mathrm{sec}, \gamma=1.87$. The wave field parameters are obtained with the following values of the initial data:

$$
f(t)=A t \cdot e-{ }^{s t}, A=1, s=1, k=0.025, h=0.05,\left|x_{2}^{0}\right|=50 h .
$$

Here $A$ is a constant factor, the parameter $s$ characterizes the velocity of the external load change. Since the investigated body has free boundaries $x_{2}= \pm 100 h$ and singularities $\left|x_{2}\right|=x_{2}^{0}=50 h$ on the boundary $x_{1}=100 h$, then over time, overlapping reflection waves (diffracted) determine the complex nature of the manifestation in it of the velocities of displacements, deformations and stresses.

The types of waves determining the stress of body points are schematized on Fig. 1.The perturbed region $a$ whose leading edge is line 1 , is determined by given load $f(t)$, varying with time. The corner points of the strip and singular points of the type $E$ are sources of the perturbation, causing both longitudinal (regions $c, e$ and $g$ ) and transverse (regions $b, d$ and $g$ ) waves.

The study of stability has shown that the grid ratio $k / h$ equaled 0.5 , provides stable results for a sufficiently large time interval under multiple reflections and diffractions of waves. In fact, the calculation was carried out to $t=$ $600 \mathrm{k}$. When calculating at any moment of time $t$, all boundary conditions are fulfilled exactly at both the corner and the singular points. This circumstance, unlike many approximate methods, ensures the reliability of the solutions obtained and the corresponding results.

The results of calculations are presented in the form of graphs for the characteristic points of the rectangular strip as a function of time $t$, and also in the form of a surface characterizing the distribution of the tensor component of stress $p+q$ in the investigated region.

Because of the symmetry of the fixation conditions and the nature of the loading, the required parameters $v_{1}, p, q$ are even and $v_{2}, \tau$ are odd with respect to the axis $x_{2}=0$. In this connection, Figs. 2-4 show the results of cal- 
culations only for positive values $x_{2}$. They show the complex character of the distribution of the velocities $v_{1}, v_{2}$ and stresses $p, q, \tau$ in the two-dimensional region. The symmetry of these functions is confirmed by calculations, that indicates the correctness of the count and the reliability of the results obtained.

The change of normal stresses $p+q, p-q$ in time on the interval $t \in[0 ; 600 \mathrm{k}]$ at six fixed observation points $-1\left(x_{1}=10 \cdot h, x_{2}=20 \cdot h\right), 2\left(x_{1}=10 \cdot h, x_{2}=50 \cdot h\right), 3\left(x_{1}=\right.$ $\left.=10 \cdot h, x_{2}=80 h\right), 4\left(x_{1}=90 \cdot h, x_{2}=20 \cdot h\right), 5\left(x_{1}=90 \cdot h\right.$, $\left.x_{2}=50 \cdot h\right), 6\left(x_{1}=90 \cdot h, x_{2}=80 \cdot h\right)$ is shown by curves in Figs. 2-3. An analysis of the behavior of normal stresses $p+q, p-q$ makes it possible to trace, to a large extent, the process of passage of various wave fronts. For small values $t$ the behavior of the curves at points $1,2,3$ is determined by the plane compression wave coming from the end-wall $x_{1}=0$. From a comparison of the oscillograms of the normal stress $p+q$ (Fig. 2), it can be seen that the stresses at points 1, 2, 3 appear simultaneously and at the initial stage of the change, on the whole, repeat profile of the loading specified by the boundary action (6). A significant increase

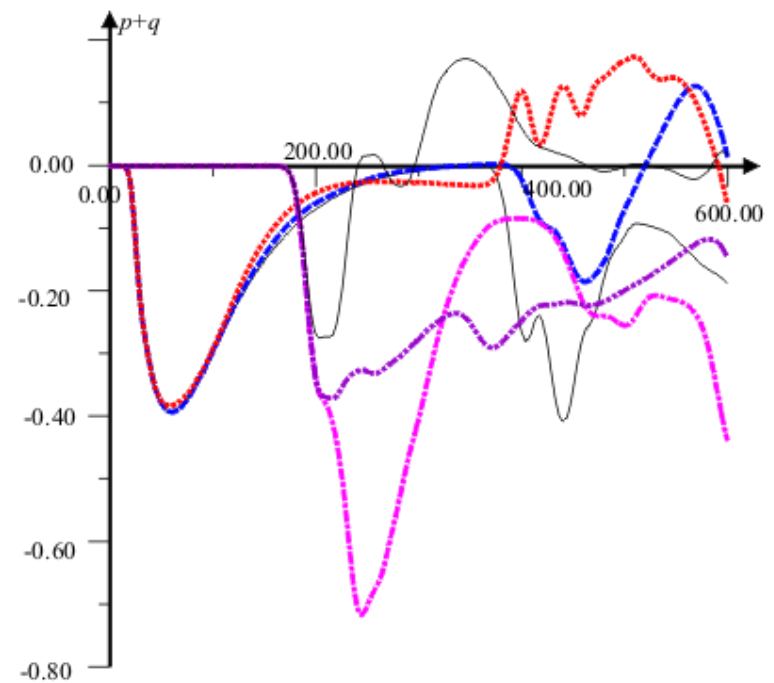

Fig. 2 Oscillogram of normal stresses $p+q$ at six $1\left(x_{1}=10 \cdot h, x_{2}=20 \cdot h\right), 2\left(x_{1}=10 \cdot h, x_{2}=50 \cdot h\right)$, $3\left(x_{1}=10 \cdot h, x_{2}=80 h\right), 4\left(x_{1}=90 \cdot h, x_{2}=20 \cdot h\right)$, $5\left(x_{1}=90 \cdot h, x_{2}=50 \cdot h\right), 6\left(x_{1}=90 \cdot h, x_{2}=80 \cdot h\right)$ fixed observation points of a rectangular area

The fixed section significantly complicates the character of the distribution of longitudinal velocities and stresses, makes them substantially two-dimensional.

Analysis of the velocities of points displacements and stresses in them shows that for a region with discontinuities in boundary conditions and for a region without discontinuity in boundary conditions far from the point of discontinuity, their distributions along the axis $x_{1} / h$ practically coincide. The differences of these parameters are observed only near the point of discontinuity, at distances equal to $6 \cdot h-8 \cdot h$ from the discontinuity point.

It should be recalled that in the developed algorithm for calculating stresses and velocities at discontinuity points in boundary conditions, the influence of an increased of the amplitude $p+q$ at points 1,4 and a decrease in the maximum compressive stresses at points 3,6 are due to the different nature of the reflection of the load wave from the fixed and free sections of the strip boundary $x_{1}=100 \mathrm{~h}$. Reflected from the free section of the strip boundary $x_{1}=100 h$ the main pulse comes at the moment $t \approx 220 \cdot k$ to point 6 , at the moment $t \approx 380 \cdot k$ to point 3 , that leads to expanding stresses. The further spreading of the main pulse in time is affected by the influence of diffraction waves. Caused by elastic waves repeatedly reflected from the plane boundaries, the normal stress $p-q$ at all points on the time interval under consideration is constantly changing its signs.

Fig. 4 shows the distribution of normal stresses $p+q$ with respect to the coordinates $x_{1} / h$ and $x_{2} / h$ at moment of time $t=300 \cdot k$. Here, the concentration of the compressive stresses is observed near the corner point $R\left(x_{1}=0, x_{2}=100 h\right)$ of the strip and the singular point $E$.

The maximum of normal stresses $p+q=-1.698$ is reached near the singular point $E$ on the boundary $x_{1}=100 \mathrm{~h}$. The level of stress concentration is a function of time and reaches different values at different moments of time.

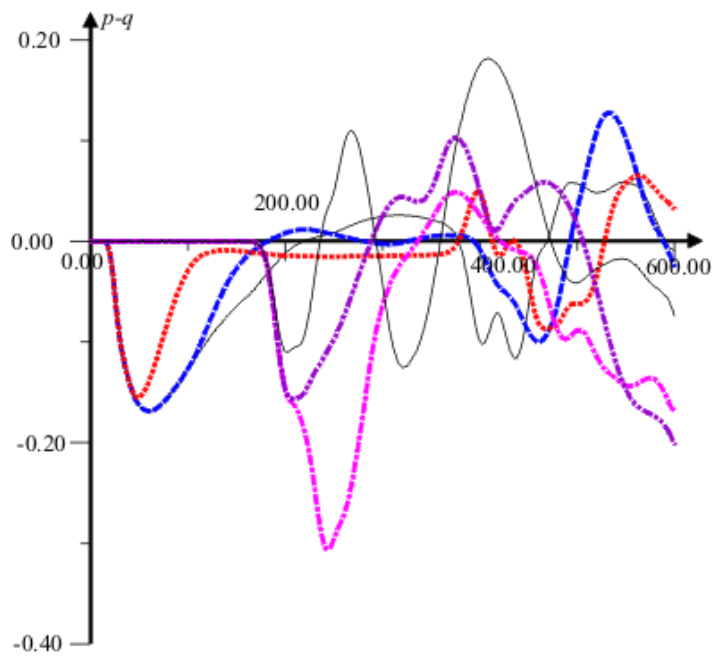

Fig. 3 Oscillogram of normal stresses $p-q$ at six $1\left(x_{1}=10 \cdot h, x_{2}=20 \cdot h\right), 2\left(x_{1}=10 \cdot h, x_{2}=50 \cdot h\right)$, $3\left(x_{1}=10 \cdot h, x_{2}=80 h\right), 4\left(x_{1}=90 \cdot h, x_{2}=20 \cdot h\right)$, $5\left(x_{1}=90 \cdot h, x_{2}=50 \cdot h\right), 6\left(x_{1}=90 \cdot h, x_{2}=80 \cdot h\right)$ fixed observation points of a rectangular area.

number of points of the investigated region is taken into account, which, it seems to us, contributes to an increase in the reliability of the results.

The developed technique can be used to investigate dynamic stresses with spasmodic change in the boundary conditions that are as close as possible to the actual types of fastening of a number of engineering structures. The developed numerical algorithm and the program of calculations in language Fortran can be used in calculations for dynamic strength of modern engineering constructions containing rectangular contour plates in the presence in them discontinuities in boundary conditions, holes, cutouts, inclusions of rectangular shape, in problems of general engineering, geophysics, defectoscopy, seismic prospecting of minerals, non-destructive testing of structures and other similar technical fields. 


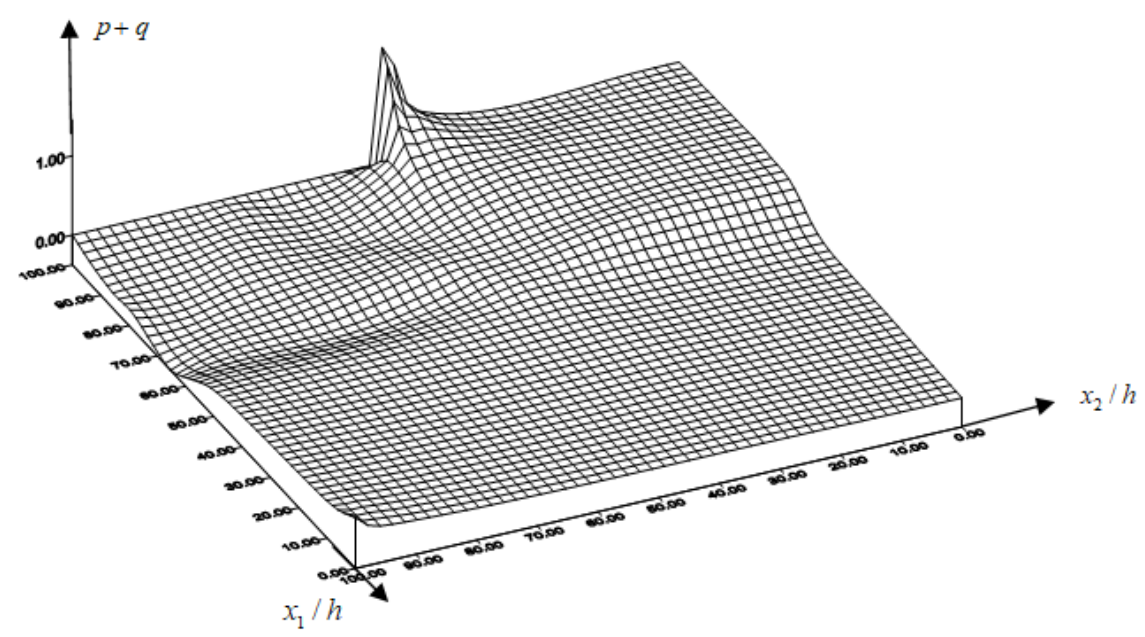

Fig. 4 Distribution of normal stresses $p+q$ on coordinates $x_{1} / h$ and $x_{2} / h$ at moment of time $t=300 \cdot k$

\section{Conclusions}

As a result of the research carried out, it can be concluded that the developed technique can be used to study dynamic stresses when jump-like change of the boundary conditions, that are as close as possible to the actual types of fastening of a number of engineering structures.

\section{References}

1. Kukudzhanov, V. N. 2011. Deformation, damage and destruction of media and materials with defects and with structures of defects, Bulletin of N.I. Lobachevsky Nizhny Novgorod University 4(4):1557-1558. (in Russian).

2. Alexeeva, L. A; Sarsenov, B. T. 2015. Mathematical model of massive dynamics in the neighborhood of 1 disturbance focus, AIP Conference Proceedings 1676: 020067.

httsp:// doi.org/10.1063/1.4930481.

3. Israilov, M. Sh. 2011. Reduction of boundary value problems of dynamic elasticity to scalar problems for wave potentials in curvilinear coordinates, Mechanics of Solids 46 (1): 104-108.

https://doi.org/10.3103/S002565441101016X.

4. Mamaev, Sh. M.; Borasheva, N. T. 2015. Investigation of dynamic perturbations propagation in composite rods by the grid-characteristic method, Izvestiya NAS RK 3 (301): 145 - 154.

5. Ashirbayev, N.; Ashirbayeva, Zh.; Sultanbek, T.; Bekmoldayeva, R. 2016. Modeling and solving the two-dimensional non-stationary problem in an elastic body with a rectangular hole, AIP Conference Proceedings 1759: 020078. https:// doi.org/10.1063/1.4959692.

6. Dolotov, M. V.; Kill', Y. D.; Limonchenko, Yu. G. 2015. Nonsymmetric tangent load on a boundary of an elastic half-space, Applied Mathematics and Mechanics 79: 264-272. (in Russian).

7. Erzhanov, Zh. S.; Karimbayev, T. D.; Bayteliyev, T. B. 1998. Waves of stresses in homogeneous and inhomogeneous media. - Almaty: Gylym, 142 p. (in Russian).
8. Clifton, R. J. 1967. A difference method for plane problems in dynamic elasticity, Quart. Appl. Math.25 (1): 97116. https://doi.org/10.1090/qam/216804.

9. Ashirbayev, N. K. 2010. Numerical solution of plane problem with discontinuities in boundary conditions, Bulletin of AbayKazNPU, series "Physical and Mathematical sciences" 29 (1): 50-54. (in Russian).

N. Ashirbayev, Zh.Ashirbayeva, G. Sarsenova,

R. Bekmoldayeva, Zh. Nurmaganbetova, P. Duisebayeva

\section{INVESTIGATION OF WAVE PROCESSES IN A REC- TANGULAR REGION WITH DISCONTINUOUS BOUNDARY CONDITIONS}

\section{S u m mary}

In the work in linear formulation the problem of propagation of nonstationary stress waves in an elastic single-supported construction, which is a rectangular strip, is solved. Formulated in terms of stresses and velocities the mixed problem is modeled numerically by means of an explicit difference scheme of through counting, based on the method of spatial characteristics. The wave process is caused by applying of an external dynamic load on the front boundary of the rectangular region, and the lateral boundaries of the region are stress-free. At the lower boundary of the rectangular region, inhomogeneous boundary conditions are given, where the middle part of the boundary is rigidly fixed, and its lateral sections are free of stresses. Namely at the points of their conjugation, where the boundary conditions change jump-like, the method is proposed for obtaining resolving equations for finding required functions. The concentration of dynamic stresses in the neighborhood of discontinuity of boundary conditions is investigated. The results of the study are brought to numerical solution.

Key words: velocity, stress, load, discontinuity, plane deformation, concentration of stresses, numerical solution. 